\title{
1 BCG-induced T cells shape Mycobacterium tuberculosis infection before reducing the
}

\section{2 bacterial burden ${ }^{1}$}

3

4 Jared L. Delahaye*, ${ }^{*}$, Benjamin H. Gern*, Sara B. Cohen*, Courtney R. Plumlee*, Shahin

5 Shafiani*, Michael Y. Gerner ${ }^{\dagger}$, Kevin B. Urdahl ${ }^{*}, \dagger, \downarrow, \S$

6

7 Running title: BCG shapes early immunity to Mtb

8

9 *Seattle Children's Research Institute, Seattle, WA 98109, USA

$10 †$ Department of Immunology, University of Washington School of Medicine, Seattle, WA

1198109, USA

12 \$Department of Pediatrics, University of Washington School of Medicine, Seattle, WA 98109,

13 USA

14 §Corresponding author:

15 Phone: 206-884-3231 Fax: 206-884-3104 kevin.urdah1@seattlechildrens.org

\footnotetext{
${ }^{1}$ This study was supported by the NIH grants 1R01AI134246 (K.B.U.), 1R01AI076327 (K.B.U.), U19AI135976 (K.B.U.), 1K22AI108628-01A1 (M.Y.G.), and T32GM007270-42 (J.L.D.).
} 


\section{Abstract}

17 Growing evidence suggests the outcome of Mycobacterium tuberculosis (Mtb) infection is

18 established rapidly after exposure, but how the current tuberculosis vaccine, BCG, impacts early

19 immunity is poorly understood. Here we found that murine BCG immunization promotes a

20 dramatic shift in infected cell types. While alveolar macrophages (AM) are the major infected

21 cell for the first two weeks in unimmunized animals, BCG promotes the accelerated recruitment

22 and infection of lung infiltrating phagocytes. Interestingly, this shift is dependent on CD4 T

23 cells, yet does not require intrinsic recognition of antigen presented by infected AM. Mtb-

24 specific $\mathrm{T}$ cells are first activated in lung regions devoid of infected cells, and these events

25 precede vaccine-induced reduction of the bacterial burden, which occurs only after the co-

26 localization of T cells and infected cells. Understanding how BCG alters early immune responses

27 to Mtb provides new avenues to improve upon the immunity it confers. 
Introduction

29 Bacillus Calmette-Guerin (BCG), the current tuberculosis (TB) vaccine, is effective at

30 preventing disseminated disease in infants and young children (1). However, in most settings it

31 provides little or no protection against adult pulmonary TB, the transmissible form of disease (2).

32 Thus, despite widespread BCG immunization for nearly a century, Mycobacterium tuberculosis

33 (Mtb) kills over 1.5 million people every year, more than any other single infectious agent (3). A

34 better TB vaccine is urgently needed, but attaining this goal has been surprisingly difficult (4).

35 Furthermore, because BCG reduces childhood mortality, a new vaccine will likely be added to a

36 regimen that includes $\mathrm{BCG}$, rather than replace it (5). To develop a strategy that builds upon

37 BCG-mediated protection, we must first understand how BCG shapes immunity to Mtb,

38 especially during early stages of infection when protective immunity is established.

39 In mice, pulmonary Mtb burdens are equivalent between BCG-immunized and control mice until

40 two weeks after infection (6). The failure of BCG to impact the Mtb burden during the first two

41 weeks of infection has been attributed to the delayed arrival of T cells in the lung (7). However,

42 BCG-specific T cells have been shown to be present in the lungs (8) of immunized mice even

43 prior to $\mathrm{Mtb}$ challenge, indicating that impaired $\mathrm{T}$ cell recruitment cannot fully account for the

44 inability of BCG to induce early protection.

46 In this study, we utilized the mouse model to investigate the impact of BCG on the early immune

47 response to Mtb infection. Our findings reveal unexpected roles for CD4 T cells in: 1)

48 accelerating the translocation of Mtb-infected alveolar macrophages (AM) into the lung

49 interstitium; 2) recruiting monocyte-derived macrophages; and 3) promoting the early transfer of

50 Mtb from AM to other phagocytes. 


\section{Materials and Methods}

52 Mice

$53 \mathrm{C} 57 \mathrm{BL} / 6$ and $\mathrm{MHCII}^{--}$mice were purchased from Jackson Laboratories (Bar Harbor, ME). All

54 mice were housed in specific pathogen-free conditions at Seattle Children's Research Institute

55 (SCRI). Experiments were performed in compliance with the SCRI Animal Care and Use

56 Committee. Both male and female mice between the ages of 8-12 weeks were used.

57

58 BCG immunization

59 BCG-Pasteur was cultured in Middlebrook $7 \mathrm{H} 9$ broth at $37^{\circ} \mathrm{C}$ to an OD of $0.2-0.5$. Bacteria was

60 diluted in PBS and $10^{6} \mathrm{CFU}$ in 200ul was injected subcutaneously. After immunization, mice

61 were rested for 8 weeks prior to Mtb infection.

62

63 Aerosol Infections

64 Infections were performed with wildtype H37Rv Mtb or H37Rv transformed with an mCherry

65 reporter plasmid (9). Mice were enclosed in a Glas-Col aerosol infection chamber and 50-100

66 CFU were deposited directly into the lungs.

67

68 Intratracheal and intravenous labeling

69 For intratracheal labeling, 30min prior to sacrifice, mice were anesthetized with $25 \%$ isoflurane

70 in propylene glycol (Fisher Scientific) and 0.25ug of CD45.2 PE-Cy7 in 50ul of PBS was

71 pipetted into the airway. For intravenous (i.v.) labeling, mice were anesthetized as above and

72 infused with CD45.2 PE 10 min prior to sacrifice. 
$74 \quad$ Lung cell isolation and antibody staining

75 Mouse lungs were homogenized in HEPES buffer with Liberase Blendzyme 3 (70ug/ml; Roche)

76 and DNaseI (30ug/ml; Sigma-Aldrich) using a gentleMacs dissociator (Miltenyi Biotec). Lungs

77 were incubated at $37^{\circ} \mathrm{C}$ for $30 \mathrm{~min}$ and then further homogenized with the gentleMacs. Cells

78 were filtered through a 70um cell strainer and resuspended in RBC lysis buffer (Thermo) prior to

79 a PBS wash. Cells were next incubated with 50ul Zombie Aqua viability dye (BioLegend) for

$8010 \mathrm{~min}$ at room temperature. Viability dye was quenched with $100 \mathrm{ul}$ of antibody cocktail in $50 \%$

81 FACS buffer (PBS containing 2.5\% FBS and 0.1\% NaN3)/50\% Fc block buffer. Staining was

82 performed for $20 \mathrm{~min}$ at $4^{\circ} \mathrm{C}$. Cells were washed with FACS buffer and fixed with $2 \%$

83 paraformaldehyde for $1 \mathrm{hr}$ prior to analysis on an LSRII flow cytometer (BD Biosciences). When

84 stain sets contained tetramers, staining was performed for $1 \mathrm{hr}$ at room temperature. Ag85B and

85 TB10.4 tetramers were obtained from the NIH Tetramer Core Facility.

$87 \quad$ Imaging

88 Mice were infected with H37Rv Mtb-mCherry and sacrificed at D10 and D14. Lungs were

89 excised and submerged in BD Cytofix fixative solution diluted 1:3 with PBS for $24 \mathrm{hr}$ at $4^{\circ} \mathrm{C}$.

90 Lungs were washed $2 \mathrm{x}$ in PBS and dehydrated in $30 \%$ sucrose for $24 \mathrm{hr}$ prior to OCT embedding

91 and rapid freezing in a methylbutane-dry ice slurry. 20um sections were stained overnight at

92 room temperature and coverslipped with Fluoromount G mounting media (Southern Biotec).

93 Images were acquired on a Leica SP8X confocal microscope, compensated for fluorophore

94 spillover using LAS X (Leica), and analyzed with Imaris (Bitplane) and FlowJo (10).

95

96 T cell depletion 
97 Mice were intraperitoneally injected with 400ug anti-CD4 GK1.5 or anti-CD8 2.43 (BioXcell) in

$98 \quad$ PBS at D-1, D4, and D10 relative to infection.

99 Bone marrow chimeras

100 WT CD45.1/2 F1 mice were irradiated with 1000 rads and reconstituted with a 1:1 mixture of

101 CD3-depleted (Miltenyi Biotec) CD45.1 B6.SJL:CD45.2 $\mathrm{MHCII}^{-/-}$bone marrow. At D56 post-

102 reconstitution, mice were immunized with BCG.

103

104 Th1 polarization and adoptive transfers

105 CD4 T cells from ESAT-6-specific (C7) (11) CD90.1 ${ }^{+}$and OVA-specific (OTII) CD45.1 ${ }^{+}$TCR

106 transgenic mice were negatively enriched from spleens using EasySep magnetic microbeads

107 (STEMCELL). T cells were Th1 polarized as follows: $1.6 \times 10^{6}$ transgenic T cells were cultured

108 with $8.3 \times 10^{6}$ irradiated CD3 $^{-}$splenocytes. $5 \mu \mathrm{g} / \mathrm{ml}$ of ESAT-6 or OVA peptide, $10 \mathrm{ng} / \mathrm{ml} \mathrm{IL-12,}$

109 and $10 \mu \mathrm{g} / \mathrm{ml}$ of anti-IL-4 antibody (R\&D Systems) were added at D0. At D3, cells were split

110 1:2, and $10 \mathrm{ng} / \mathrm{ml} \mathrm{IL}-12$ was added (R\&D Systems). On D5, Th1 cells were i.v. injected into B6

111 CD45.2 $2^{+}$mice infected with Mtb 35 days prior. 


\section{Results and Discussion}

\section{BCG vaccination promotes Mtb egress from AM early in infection.}

114 To understand the effects of BCG immunization on early Mtb infection, we examined the

115 pulmonary Mtb burdens in BCG-immunized and control mice. Consistent with prior reports (6,

116 7), lung burdens rose similarly in both groups through two weeks (Fig. 1A). At D15, the Mtb

117 burden in the immunized group began to diverge and was reduced by one log by D21. These

118 findings are consistent with the idea that BCG-induced immunity is not initiated until the third

119 week of Mtb infection.

120

121 We recently found that Mtb first infects AM before disseminating to other cells including

122 neutrophils (PMN) and monocyte-derived macrophages (MDM) (12). As tissue-resident and

123 recruited phagocytes have been shown to differ in their capacity to curb Mtb replication $(13,14)$,

124 we next asked whether immunization alters the proportions of cell types that harbor infection.

125 Consistent with the similar Mtb burdens at D14, the numbers of cells harboring fluorescent Mtb

126 (Mtb-mCherry) were also similar in each group (Fig. 1B). Surprisingly, even at this early phase,

127 we observed a dramatic shift in the composition of infected cells. At D14, the proportion of Mtb-

128 infected AM was significantly reduced in immunized animals compared to controls, with a

129 corresponding increase in infected PMN and MDM (Fig. 1C-D). We confirmed these findings

130 using confocal microscopy and quantitative histocytometry (10), wherein most Mtb was within

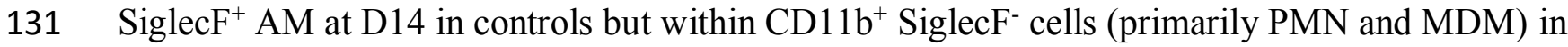
132 immunized mice (Fig. 1E-F). As Mtb dissemination to PMN and MDM requires translocation of

133 infected AM to the lung interstitium (12), we next assessed whether this translocation was

134 accelerated in immunized mice. Indeed, intratracheal antibody administration, which specifically 
135 labels alveolar-localized cells (12), revealed significantly increased interstitial localization (labelnegative) of infected AM in immunized mice at D14 (Fig. 1G). Finally, immunization

137 significantly enhanced MDM recruitment to the lung at D14 (Fig. 1H), which was not observed

138 at earlier time points or in Mtb-naïve mice (Supplemental Fig. 1A), suggesting that the

139 accelerated recruitment of MDM in immunized mice begins between D10 and D14. Thus,

140 although BCG does not impact the pulmonary Mtb burden in the first 2 weeks of infection, it

141 accelerates the translocation of infected AM from alveoli to the lung interstitium, MDM

142 recruitment, and Mtb dissemination to PMN and MDM.

144 BCG accelerates the recruitment of antigen-specific T cells to the lung following Mtb

\section{5 infection.}

146 This unexpected impact of BCG on the early dynamics of infection led us to next investigate

147 how immunization affects the kinetics of $\mathrm{T}$ cell recruitment to the lung. Before infection, 148 antigen-specific CD4 (Ag85B) and CD8 (TB10.4) T cells could be identified in lung cell 149 suspensions of immunized mice (Fig. 2A-C). Although $25 \%$ of the Ag85B-specific cells were 150 located in the lung parenchyma (as evidenced by their failure to stain with i.v. CD45 antibody), 151 virtually all of the TB10.4-specific cells resided in the vasculature (Fig. 2D). Following

152 infection, immunized mice had significantly more Ag85B-specific and TB10.4-specific cells in 153 the lung parenchyma than controls as early as D10; by D14 they contained $>5$-fold more (Fig.

154 2B-C). Thus, BCG induces a small population of lung-resident Mtb-specific CD4 T cells prior to 155 infection. After infection, BCG accelerates the pulmonary recruitment of both CD4 and CD8 156 Mtb-specific T cells, even before impacting the Mtb burden. 
CD4 T cells are required for the accelerated transfer of Mtb from AM to recruited phagocytes.

Given the presence of lung-resident Mtb-specific T cells in immunized mice prior to infection,

161 we next determined whether T cells play a role in the accelerated transfer of Mtb from AM to

162 other myeloid cells. CD4 or CD8 T cells were depleted from immunized mice beginning 1 day

163 prior to Mtb-mCherry infection and lung cells were assessed at D14 (Supplemental Fig. 1B-C).

164 In the absence of CD4 T cells, the accelerated transfer of Mtb from AM to PMN and MDM was

165 partially reversed, whereas CD8 T cell depletion had no effect (Fig. 3A). Interestingly, the

166 accelerated MDM recruitment (Fig. 1H) was also abolished by CD4 depletion (Fig. 3B). We next

167 investigated whether direct recognition of Mtb-infected cells by CD4 $\mathrm{T}$ cells was required for the

168 early dissemination out of the AM niche and whether $\mathrm{MHCII}^{-/-} \mathrm{AM}$, which cannot present

169 antigen to CD4 T cells, would retain Mtb longer than WT AM. WT:MHCII ${ }^{-/-}$mixed bone

170 marrow chimeras were generated, BCG immunized, and infected with Mtb-mCherry. At D14,

171 BCG induced the accelerated transfer of Mtb from AM to other myeloid cells irrespective of

172 intrinsic MHCII expression (Fig. 3C). Taken together, BCG-induced CD4 T cells promote the

173 early transfer of Mtb from AM to other myeloid cells in a process that does not require direct

174 cognate interactions between T cells and Mtb-infected AM. Our finding that CD4 T cells

175 promote MDM recruitment to the lung, thereby providing new bacterial targets, may help

176 explain the increased proportion of infected MDM in immunized animals. This recruitment

177 likely relates to T cell production of cytokines, such as IFN-gamma and TNF, which are known

178 to trigger the release of chemokines that act on MDM, i.e., CCL2 and CXCL10 (15). 
181 We next investigated the site of CD4 T cell activation during early Mtb infection using phospho-

182 S6 (pS6) as a marker of TCR signaling, which is rapidly induced by TCR engagement, peaking

183 at $4 \mathrm{~h}$ and resolving within $24 \mathrm{~h}$ (16). To confirm that pS6 expression by T cells is TCR-

184 dependent in the context of Mtb-infected lungs, we demonstrated that pS6 was robustly expressed by adoptively transferred TCR transgenic Mtb-specific (ESAT-6; C7) CD4 T cells

186 compared to irrelevant TCR transgenic T cells (OVA-specific) (Supplemental Fig. 2A). We next

187 performed quantitative histocytometry to assess the intrapulmonary location of pS6 expression

188 by CD4 T cells. At D10, there were significantly more $\mathrm{pS}^{+} \mathrm{CD} 4 \mathrm{~T}$ cells in the lungs of 189 immunized mice compared to controls (Fig. 4A-B, Supplemental Fig. 2F). Surprisingly, few of

190 these cells were located near infected cells (Fig. 4A, 4D, Supplemental Fig. 2B-E). Thus,

191 although BCG induces early T cell recruitment and activation, at D10 this occurs primarily in

192 uninfected areas of the lung, which may be due to Mtb antigenic export from infected to

193 uninfected antigen-presenting cells (17). Taken together, the activation of BCG-induced CD4 T

194 cells, which occurs distal to sites of infection, shapes immunity to Mtb challenge earlier than

195 previously appreciated by facilitating the pulmonary recruitment of MDM and accelerating the

196 transfer of Mtb from AM to other myeloid cells. This transfer likely influences the ability of the

197 BCG-immunized host to control Mtb, as prior studies have shown that tissue-resident vs.

198 recruited macrophages differ profoundly in their capacity to control Mtb replication $(13,14)$.

199 Future studies are needed to elucidate the overall impact on protection because the settings in

200 which distinct macrophage types mediate enhanced immunity remain unclear.

201

202 Interestingly, BCG-induced CD4 T cells only begin to curb Mtb replication at D14, when they 203 finally co-localize with cells harboring Mtb, as evidenced by the identification of many $\mathrm{pS}^{+} \mathrm{T}$ 
204 cells at sites of infection compared to controls (Fig. 4C-D). This is consistent with the finding

205 that optimal immunity against Mtb requires direct interactions between antigen-specific CD4 T

206 cells and Mtb-infected cells (21). Why do T cells and Mtb-infected cells not co-localize earlier?

207 The AM is the first cell type to become infected and remains the primary infected cell type for at

208 least a week (12). During this time, the immune system appears largely unaware of the looming

209 threat, as few MDM or PMN are recruited to the lung. The recent finding that AM infection is

210 non-inflammatory and poorly induces chemokines may help explain the covert nature of early

211 infection (Rothchild, A.C. et al. 2019. bioRxiv: 520791). Furthermore, the replication and spread

212 within the AM population, a process associated with cell death of infected macrophages and

213 phagocytosis by other macrophages, likely involves apoptosis, as necrotic cell death is associated

214 with chemokine release and recruitment of MDM/PMN (19). Perhaps vaccine-induced T cells

215 that express receptors for apoptotic "find-me" signals could co-localize with infected AM and

216 exhibit earlier Mtb control compared to BCG-induced T cells, which may not express such

217 receptors (20). Together, these results further our understanding of the features of pulmonary

218 Mtb dissemination in the context of BCG, which could aid rational vaccine design to effectively

219 complement BCG. 
bioRxiv preprint doi: https://doi.org/10.1101/590554; this version posted April 4, 2019. The copyright holder for this preprint (which was not certified by peer review) is the author/funder, who has granted bioRxiv a license to display the preprint in perpetuity. It is made available under aCC-BY-NC-ND 4.0 International license.

\section{Acknowledgements}

221 We thank P. Andersen, J. Woodworth, and R. Mortensen for critical feedback of the manuscript

222 as well as the flow cytometry core and vivarium staff for technical assistance. 


\section{References}

224 1. Trunz, B. B., P. Fine, and C. Dye. 2006. Effect of BCG vaccination on childhood

2. Mangtani, P., I. Abubakar, C. Ariti, R. Beynon, L. Pimpin, P. E. Fine, L. C. Rodrigues, P. tuberculous meningitis and miliary tuberculosis worldwide: a meta-analysis and assessment of cost-effectiveness. Lancet 367: 1173-1180.

G. Smith, M. Lipman, P. F. Whiting, and J. A. Sterne. 2014. Protection by BCG vaccine against tuberculosis: a systematic review of randomized controlled trials. Clin Infect Dis 58: 470-480.

3. World Health Organization. 2018. Global tuberculosis report 2018. World Health Organization, Geneva.

4. Tameris, M. D., M. Hatherill, B. S. Landry, T. J. Scriba, M. A. Snowden, S. Lockhart, J. E. Shea, J. B. McClain, G. D. Hussey, W. A. Hanekom, H. Mahomed, H. McShane, and M. A. T. S. Team. 2013. Safety and efficacy of MVA85A, a new tuberculosis vaccine, in infants previously vaccinated with BCG: a randomised, placebo-controlled phase $2 \mathrm{~b}$ trial. Lancet 381: 1021-1028.

5. Roth, A. E., L. G. Stensballe, M. L. Garly, and P. Aaby. 2006. Beneficial non-targeted effects of BCG--ethical implications for the coming introduction of new TB vaccines. Tuberculosis (Edinb) 86: 397-403.

6. Mollenkopf, H. J., M. Kursar, and S. H. Kaufmann. 2004. Immune response to postprimary tuberculosis in mice: Mycobacterium tuberculosis and Mycobacterium bovis bacille Calmette-Guerin induce equal protection. J Infect Dis 190: 588-597.

7. Ronan, E. O., L. N. Lee, P. C. Beverley, and E. Z. Tchilian. 2009. Immunization of mice with a recombinant adenovirus vaccine inhibits the early growth of Mycobacterium tuberculosis after infection. PLoS One 4: e8235.

8. Santosuosso, M., S. McCormick, X. Zhang, A. Zganiacz, and Z. Xing. 2006. Intranasal boosting with an adenovirus-vectored vaccine markedly enhances protection by parenteral Mycobacterium bovis BCG immunization against pulmonary tuberculosis. Infect Immun 74: 4634-4643.

9. Cosma, C. L., O. Humbert, and L. Ramakrishnan. 2004. Superinfecting mycobacteria home to established tuberculous granulomas. Nat Immunol 5: 828-835.

10. Gerner, M. Y., W. Kastenmuller, I. Ifrim, J. Kabat, and R. N. Germain. 2012. Histocytometry: a method for highly multiplex quantitative tissue imaging analysis applied to dendritic cell subset microanatomy in lymph nodes. Immunity 37: 364-376.

11. Gallegos, A. M., E. G. Pamer, and M. S. Glickman. 2008. Delayed protection by ESAT6-specific effector CD4+ T cells after airborne M. tuberculosis infection. J Exp Med 205: 2359-2368.

12. Cohen, S. B., B. H. Gern, J. L. Delahaye, K. N. Adams, C. R. Plumlee, J. K. Winkler, D. R. Sherman, M. Y. Gerner, and K. B. Urdahl. 2018. Alveolar Macrophages Provide an Early Mycobacterium tuberculosis Niche and Initiate Dissemination. Cell Host Microbe 24: 439-446 e434.

13. Huang, L., E. V. Nazarova, S. Tan, Y. Liu, and D. G. Russell. 2018. Growth of Mycobacterium tuberculosis in vivo segregates with host macrophage metabolism and ontogeny. J Exp Med 215: 1135-1152. 
14. Cambier, C. J., K. K. Takaki, R. P. Larson, R. E. Hernandez, D. M. Tobin, K. B. Urdahl, C. L. Cosma, and L. Ramakrishnan. 2014. Mycobacteria manipulate macrophage recruitment through coordinated use of membrane lipids. Nature 505: 218-222.

15. Murray, H. W., A. D. Luster, H. Zheng, and X. Ma. 2017. Gamma Interferon-Regulated Chemokines in Leishmania donovani Infection in the Liver. Infect Immun 85.

16. Sauer, S., L. Bruno, A. Hertweck, D. Finlay, M. Leleu, M. Spivakov, Z. A. Knight, B. S. Cobb, D. Cantrell, E. O'Connor, K. M. Shokat, A. G. Fisher, and M. Merkenschlager. 2008. T cell receptor signaling controls Foxp3 expression via PI3K, Akt, and mTOR. Proc Natl Acad Sci U S A 105: 7797-7802.

17. Srivastava, S., P. S. Grace, and J. D. Ernst. 2016. Antigen Export Reduces Antigen Presentation and Limits T Cell Control of M. tuberculosis. Cell Host Microbe 19: 44-54.

20. Hochreiter-Hufford, A., and K. S. Ravichandran. 2013. Clearing the dead: apoptotic cell

18. Srivastava, S., and J. D. Ernst. 2013. Cutting edge: Direct recognition of infected cells by CD4 T cells is required for control of intracellular Mycobacterium tuberculosis in vivo. $J$ Immunol 191: 1016-1020.

19. Almeida, F. M., T. L. Ventura, E. P. Amaral, S. C. Ribeiro, S. D. Calixto, M. R. Manhaes, A. L. Rezende, G. S. Souzal, I. S. de Carvalho, E. C. Silva, J. A. Silva, E. C. Carvalho, A. L. Kritski, and E. B. Lasunskaia. 2017. Hypervirulent Mycobacterium tuberculosis strain triggers necrotic lung pathology associated with enhanced recruitment of neutrophils in resistant C57BL/6 mice. PLoS One 12: e0173715. sensing, recognition, engulfment, and digestion. Cold Spring Harb Perspect Biol 5: a008748.

21. Muller, A. J., O. Filipe-Santos, G. Eberl, T. Aebischer, G. F. Spath, and P. Bousso. 2012. CD4+ T cells rely on a cytokine gradient to control intracellular pathogens beyond sites of antigen presentation. Immunity 37: 147-157. 


\section{Figure legends}

293 Figure 1: BCG vaccination promotes Mtb egress from AM early in infection.

294 (A) Mtb burden in the lungs of mice that did or did not receive $B C G(n=4$

295 mice/group/timepoint). (B) Total number of $\mathrm{mCherry}^{+}$lung cells at D14 by flow cytometry $(\mathrm{n}=4-$

2965 mice/group). (C) Representative flow plot of the proportion of mCherry ${ }^{+}$cells identified as

297 CD11 $\mathrm{c}^{+}$Siglec-F $\mathrm{F}^{+}$AMs at D14. (D) Composition of mCherry ${ }^{+}$lung cells (AM: CD11c ${ }^{+}$Siglec-F ${ }^{+}$,

298 PMN: CD11b Ly6G $^{+}, \mathrm{MDM}^{+} \mathrm{CD} 11 \mathrm{~b}^{+} \mathrm{CD}^{+} 4^{+}$) at D14 by flow cytometry (n=4-5 mice/group).

299 (E) Representative images of the lung at D14 showing infected Siglec $\mathrm{F}^{+}$AM (orange arrows)

300 and infected Siglec F- CD11 $\mathrm{b}^{+}$cells (white arrows). (F) Composition of mCherry ${ }^{+}$lung cells at

301 D14 by quantitative histocytometry ( $\mathrm{n}=6-8$ infectious foci from 2 mice/group). (G) Ratio of

302 airway label positive infected AM at D14 (n=5 mice/group). (H) Number of MDM in the lung at

303 D14 by flow cytometry (n=4-5 mice/group). Single-group comparisons were performed by

304 unpaired t test. Data are presented as mean \pm SEM. $* \mathrm{p}<0.05, * * \mathrm{p}<0.01, * * * * \mathrm{p}<0.0001$. All

305 experiments were performed at least 2-3 times.

306

307 Figure 2: BCG accelerates the recruitment of antigen-specific T cells to the lung following Mtb

308 infection.

309 Time course of the number of tetramer-specific T cells in the lung. Mice received i.v. CD45

310 antibody prior to sacrifice. (A) Representative flow plots showing Ag85B-specific $\left(\mathrm{CD} 3^{+} \mathrm{CD} 4^{+}\right)$

311 and TB10.4-specific $\left(\mathrm{CD}^{+} \mathrm{CD}^{+}\right) \mathrm{T}$ cells in the lungs of control and immunized mice prior to

312 infection. The tetramer ${ }^{+}$cells in immunized mice are further gated on CD45 i.v.- to determine the

313 proportion in the lung parenchyma. Total number of i.v.' Ag85B-specific (B) and TB10.4-

314 specific (C) cells in the lungs of control and immunized mice ( $\mathrm{n}=3-5 \mathrm{mice} / \mathrm{group} / \mathrm{timepoint})$. (D) 
315 Proportion of tetramer ${ }^{+}$cells that are i.v.- in immunized mice at D0 ( $n=5$ mice/group). Single-

316 group comparisons were performed by unpaired t test. Data are presented as mean \pm SEM. *p $<$

$3170.05, * * \mathrm{p}<0.01, * * * \mathrm{p}<0.001$. All experiments were performed at least twice.

318

319 Figure 3: CD4 T cells are required for the accelerated transfer of Mtb from AM to recruited 320 phagocytes.

321 (A) Composition of mCherry ${ }^{+}$cells in control, immunized, and T cell-depleted immunized mice

322 at D14 (n=4 mice/group). (B) Total number of MDM as in (A). (C) Composition of WT (left)

323 and $\mathrm{KO}$ (right) $\mathrm{mCherry}^{+}$cells in control and immunized mixed bone marrow chimeras at D14

324 ( $n=3-4$ mice/group). Single-group comparisons were performed by unpaired t test (C) and

325 multiple-group comparisons by one-way ANOVA (A and B). Data are presented as mean \pm

326 SEM. ${ }^{*} \mathrm{p}<0.05,{ }^{* *} \mathrm{p}<0.01,{ }^{* * *} \mathrm{p}<0.001, * * * * \mathrm{p}<0.0001$. All experiments were performed at

327 least twice.

329 Figure 4: BCG-induced CD4 T cells are initially activated distal to the site of Mtb infection.

330 Quantitative histocytometry was used to identify the location of CD4 T cells (blue) and pS6 ${ }^{+}$

331 CD4 T cells (green) relative to infected cells (red) in lung sections at D10 (A) and sites of

332 infection at D14 (C). (B) Number of $\mathrm{pS}^{+} \mathrm{CD} 4 \mathrm{~T}$ cells per $\mathrm{mm}^{2}$ of lung at D10 as determined by 333 quantitative histocytometry ( $\mathrm{n}=2-3$ mice/group). (D) Number of pS6 ${ }^{+}$CD4 T cells within $80 \mu \mathrm{m}$

334 of an infected cell ( $\mathrm{n}=2$ mice/group). This cutoff was based on the limit of IFNg diffusion within 335 tissue (21). Single-group comparisons were performed by unpaired t test. Data are presented as 336 mean \pm SEM. *p $<0.05, * * p<0.01$ 
A

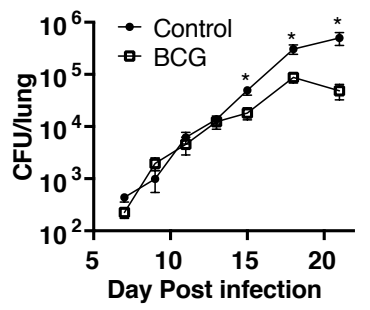

D

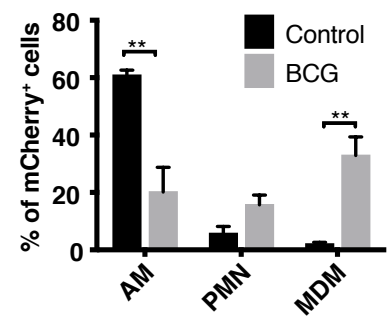

F

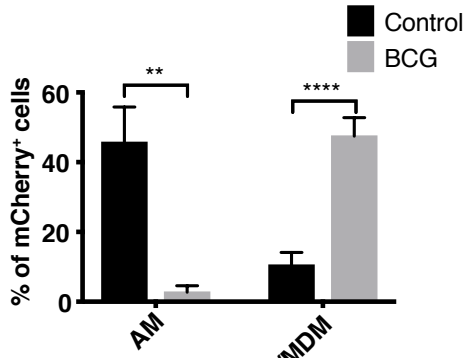

B

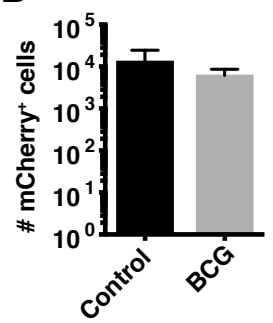

$E$
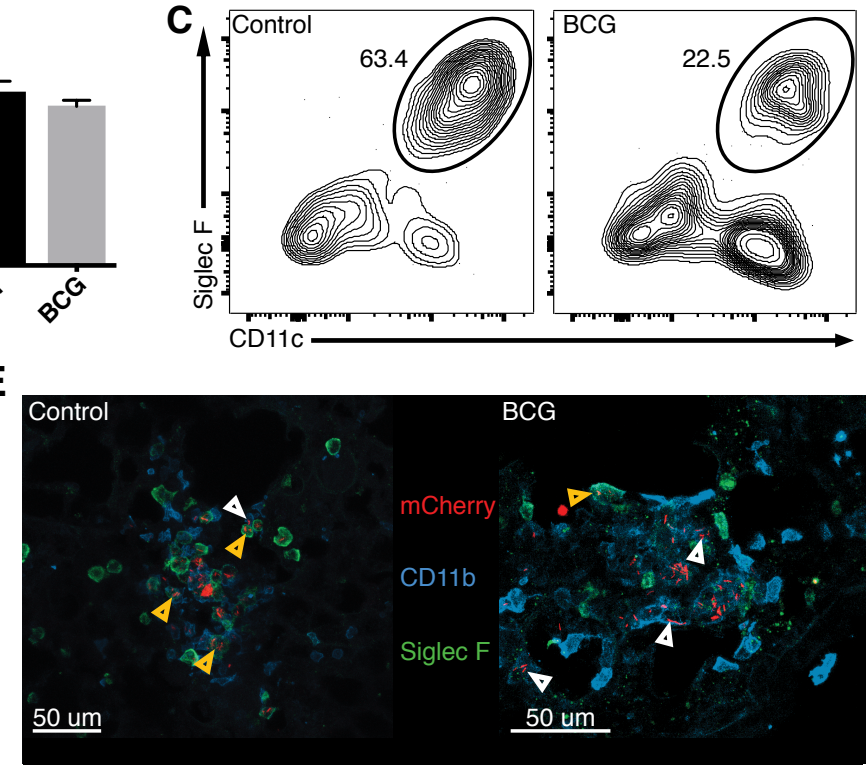

G

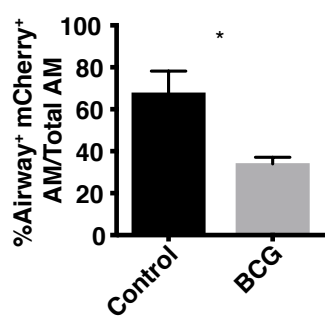

H

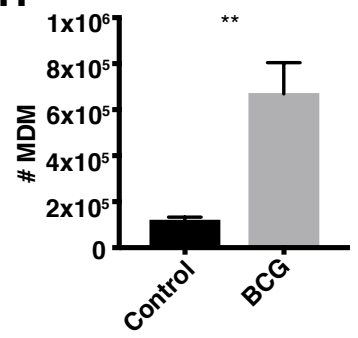


A

Control

BCG

B
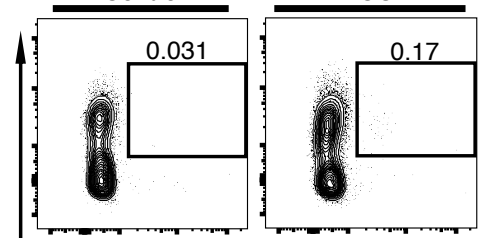

$\square$
$\square$
$\cup$

Ag85B
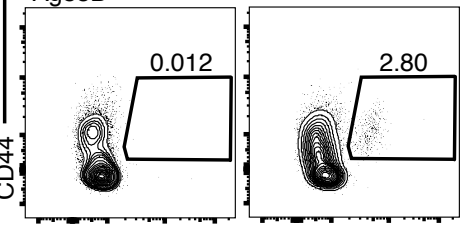

TB10.4
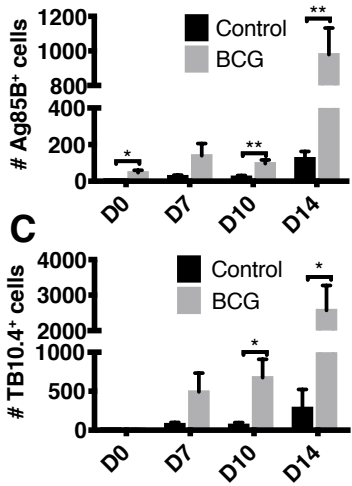

D
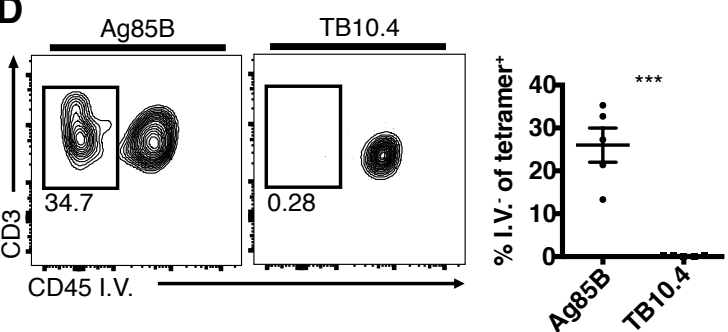

Figure 2 
A

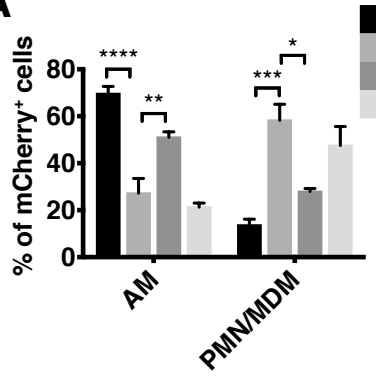

C

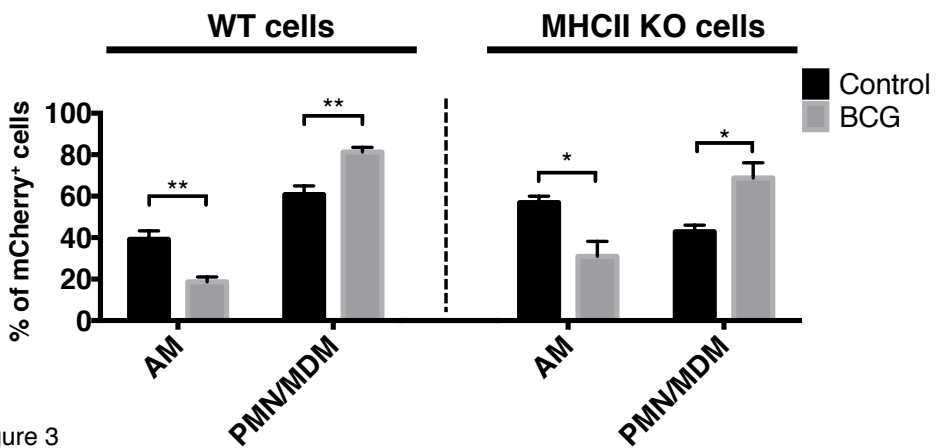

B

Control

BCG

BCG aCD4

BCG aCD8

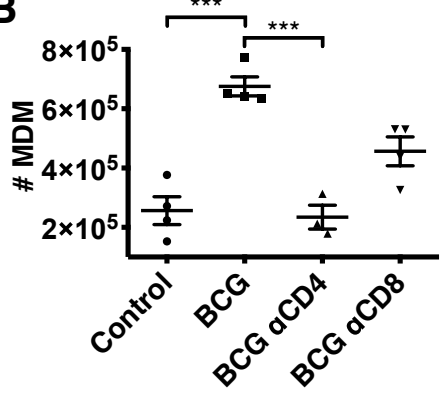

Figure 3 


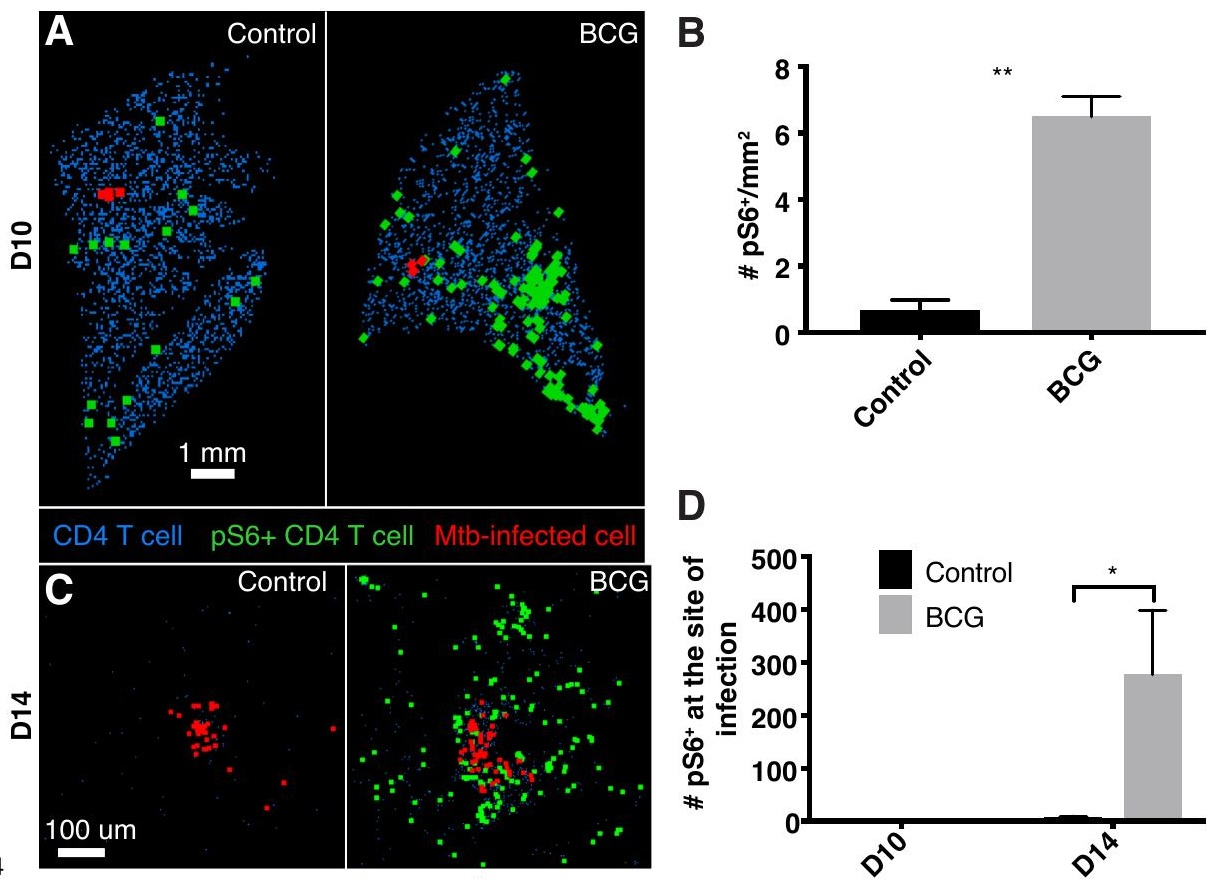

